\title{
AVALIAÇÃO DA ADIÇÃO DE AGENTES PERLITIZANTES NAS PROPRIEDADES MECÂNICAS DE UM FERRO FUNDIDO NODULAR
}

\author{
Lucas Cunha ' \\ Elídio Angioletto ${ }^{2}$ \\ Márcio Roberto Rocha ${ }^{3}$
}

\section{Resumo}

O objetivo deste trabalho foi avaliar o efeito da adição de cobre, estanho e antimônio nas microestruturas e propriedades mecânicas de um ferro fundido nodular J434 D500, além da melhor relação custo / benefício. Para o desenvolvimento, amostras com diferentes adições de $\mathrm{Cu}$, $\mathrm{Sn}$ e Sb foram fundidas e suas microestruturas foram avaliadas por microscopia óptica. As propriedades mecânicas foram avaliadas através de ensaios de tração e dureza. Os resultados indicaram o intervalo de composição adequado para se obter as propriedades estabelecidas no padrão SAE J434. Verificou-se também que para este antimônio de ferro fundido tem um efeito negativo na formação de grafite e em propriedades em quantidades superiores a $0,029 \%$ em peso. Todos estes elementos tiveram a função de aumentar a resistência mecânica do material, principalmente devido aos seus efeitos perlizantes. Entre os três elementos adicionados, Sb apresentou a melhor relação custo / benefício.

Palavras-chave: Ferro fundido nodular; Cobre; Estanho; Antimônio.

\section{EVALUATION OF THE ADDITION OF PERLITIZING AGENTS IN THE MECHANICAL PROPERTIES OF A DUCTILE CAST IRON}

\begin{abstract}
The objective of this work was to evaluate the effect of the addition of copper, tin and antimony on the microstructures and mechanical properties of a ductile cast iron J434 D500, besides the best cost / benefit ratio. For the development, samples with different additions of $\mathrm{Cu}, \mathrm{Sn}$ and $\mathrm{Sb}$ were fused and had their microstructures evaluated by optical microscopy. The mechanical properties were evaluated through tensile and hardness tests. The results indicated the range of composition adequate to obtain the properties established in SAE J434 standard. It has also been found that for this cast iron antimony has a negative effect on the formation of graphite and on properties in amounts greater than $0.029 \%$ by weight. All these elements had the function of increasing the mechanical strength of the material, mainly due to its perlizant effects. Among the three elements added, Sb presented the best cost / benefit ratio.
\end{abstract}

Keywords: Ductile cat iron; Copper; Tin; Antimony.

\section{INTRODUÇÃO}

Entre os ferros fundidos, a utilização do ferro fundido nodular ocorre devido a sua ductilidade estar aliada à boa resistência mecânica. Essas propriedades qualificam este ferro fundido para várias aplicações em determinados segmentos, tais como, produção de tubos e conexões, peças para indústria automobilística, válvulas e corpos de bombas para indústria do petróleo, componentes de máquinas sujeitos a cargas de choque e fadiga, entre outros.

A ductilidade apresentada neste tipo de ferro fundido é proveniente ao seu tipo de grafita, que se apresenta

'Laboratório de Fundição - Fundição Nobre, Nova Veneza, SC, Brasil. E-mail: lucasengtf@gmail.com

${ }^{2}$ Programa de Pós-graduação em Ciências e Engenharia de Materiais, Universidade do Extremo Sul Catarinense - UNESC, Criciúma, SC, Brasil.

${ }^{3}$ Universidade Federal de Santa Catarina - UFSC, Florianópolis, SC, Brasil.

2176-1523 (C) 2019 Associação Brasileira de Metalurgia, Materiais e Mineração. Publicado pela ABM. Este é um artigo de acesso aberto distribuído sob os termos da licença Creative Commons CC BY-NC-ND (Attribution-NonCommercial-NoDerivs) - https:// creativecommons.org/licenses/by-nc-nd/4.0\%. 
em forma esferoidal e que em matriz ferrítica conduz a ductilidade ainda maior quando comparada com a matriz perlítica. Além de possuir ductilidade e resistência mecânica, dependendo da matriz predominante, pode-se favorecer uma das propriedades mencionadas $[1,2]$.

A obtenção deste ferro fundido depende do processo de nodularização e das condições específicas do metal base, que consiste no tratamento feito no metal base com elementos químicos que favoreçam a formação de grafita esferoidal. As técnicas de nodularização mais conhecidas são, simples transferência, processo sanduíche e imersão por sino [2-4].

Com a demanda do mercado de peças que utilizam ligas em ferro fundido nodular com alta resistência mecânica, é essencial este diferencial na utilização de um elemento perlitizante de baixo custo e que apresente propriedade de acordo com as especificações da norma vigente.

O presente trabalho teve como objetivo avaliar o comportamento de três elementos perlitizantes, sendo estanho, cobre e antimônio. Avaliou-se as propriedades mecânicas e a microestrutura, afim de verificar-se qual o percentual ideal para adição e qual o elemento de liga apresenta melhor custo benefício para obtenção de um ferro fundido nodular da classe D500 da norma SAE J434 e para classes superiores.

\section{MATERIAIS E MÉTODOS}

As amostras estudadas neste trabalho, foram produzidas em fornos de indução sem núcleo com capacidade de $650 \mathrm{~kg}$ da marca Eletrotec com potência máxima de $400 \mathrm{~kW}$, utilizando liga de ferro, silício e magnésio, sendo este último o agente nodularizante. Utilizou-se o processo de tratamento conhecido como panela rotativa e adicionou-se os elementos perlitizantes na própria panela de vazamento.
Para o carregamento do forno, primeiramente foi adicionado ferro gusa, pois apresenta menor ponto de fusão, na sequência sucata de aço, grafite sintético e ferro-silício. Com o decorrer da fusão das matérias-primas, foi adicionado retorno até completar toda a capacidade do cadinho.

A amostra para análise química foi retirada com temperatura mínima de $1450^{\circ} \mathrm{C}$ com o auxílio de um cadinho e vertido em uma coquilha de cobre eletrolítico.

A composição química foi avaliada com um espectrômetro de emissão óptica (Espectro - modelo Espectromaxx).

A liga escolhida para o estudo foi a D500 da norma SAE J434 [5], cuja a especificação de composição química é apresentada na Tabela I.

Os valores mínimos e máximos descritos para cada elemento químico é aplicado para todas as classes descritas na referida norma, porém para cada classe é necessário estreitar os valores de acordo com os resultados de resistência à tração, alongamento, dureza e metalografia, conforme Tabela 2.

Com os dados da especificação da classe D500 e - processo de fabricação, foi definida uma tolerância de composição química específica para a classe D500 utilizada na empresa de acordo com a Tabela 3.

Após o ajuste da composição química segundo a Tabela 03, o metal líquido foi aquecido em uma temperatura entre 1500 e $1550^{\circ} \mathrm{C}$, foi efetuado a limpeza da superfície do metal líquido para retirada de escória decorrente do processo de refino do metal.

Antes do início do vazamento, a panela de tratamento foi aquecida com maçarico e na sequência foi adicionado a liga $\mathrm{FeSiMg} \mathrm{N} 4$ contendo de 5 a $7 \%$ de magnésio e granulometria de 6 a $12 \mathrm{~mm}$ (equivalente a I,30\% do peso do metal adicionado posteriormente nesta panela).

O processo de tratamento realizado é conhecido como panela rotativa, onde a liga é adicionada no reservatório da panela na posição vertical. Após a panela é posicionada na horizontal para receber o metal líquido. Foi adiconado I,56 kg de liga para $120 \mathrm{~kg}$ de metal base.

Tabela I. Composição química da classe D500 [5]

\begin{tabular}{ccccccc}
\hline Elementos & C & Si & Mn & P & S & Mg \\
\hline$\%$ em peso & 3,20 a 4,10 & 1,80 a 3,00 & 0,10 a I,00 & Max. 0,050 & Max. 0,035 & 0,025 a 0,060 \\
\hline
\end{tabular}

Tabela 2. Especificações da classe D500 [5]

\begin{tabular}{cc}
\hline Ensaio & Valores \\
\hline Resistência a tração mínima & $500 \mathrm{MPa}$ \\
Alongamento mínimo & $6 \%$ \\
Dureza & 187 a $229 \mathrm{HBW}$ \\
Metalografia & $80 \%$ de grafita do tipo I e ll com matriz ferrítica-perlítica ou perlítica-ferrítica e isenta de carbonetos \\
\hline
\end{tabular}

Tabela 3. Composição quimíca da classe D500 utilizado pela empresa

\begin{tabular}{ccccccc}
\hline Elementos & $\mathbf{C}$ & Si & Mn & $\mathbf{P}$ & S & Mg \\
\hline \% em peso & 3,40 a 3,80 & 2,30 a 2,80 & 0,30 a 0,50 & Máx. 0,050 & Máx. 0,035 & 0,025 a 0,060 \\
\hline
\end{tabular}


Durante a transferência do metal da panela de tratamento para a panela de vazamento, foi adicionado $0,60 \%$ de inoculante contendo silíco, cálcio e bário com peso total de $0,720 \mathrm{~kg}$, mais o elemento perlitizante de acordo com a Tabela 4.

Durante o vazamento foi medido a temperatura do metal com um pirômetro de imersão da marca Italtherm utilizando o sensor do tipo " $S$ " para manter-se em média a $1400^{\circ} \mathrm{C}$, evitando qualquer tipo de defeito metalúrgico. Após o vazamento das amostras, foi definido o tempo de resfriamento de 30 minutos de modo que esses parâmetros fossem semelhantes para as 3 fornadas testadas com a composição química base controlada.

Em cada panela de tratamento, foram vazados quatro blocos em " $Y$ " [6,7] para obtenção de 4 corpos de prova de tração, duas amostras para medição de dureza superficial e uma amostra metalográfica. Na Figura I estão indicadas as regióes de análise a partir do corpo de prova fundido.

Tabela 4. Quantidades dos elementos perlitizantes

\begin{tabular}{cccc}
\hline \multicolumn{1}{c}{ Adições } & & $\%$ & kg \\
\hline Adição de $\mathrm{Cu}$ & $1^{\circ}$ & 0,30 & 0,387 \\
& $2^{\circ}$ & 0,60 & 0,773 \\
\multirow{3}{*}{ Adição de Sb } & $3^{\circ}$ & 0,90 & 1,160 \\
& $1^{\circ}$ & 0,03 & 0,036 \\
Adição de Sn & $2^{\circ}$ & 0,06 & 0,07 \\
& $3^{\circ}$ & 0,09 & 0,109 \\
& $1^{\circ}$ & 0,03 & 0,037 \\
& $2^{\circ}$ & 0,06 & 0,074 \\
& $3^{\circ}$ & 0,09 & 0,111 \\
\hline
\end{tabular}

A medição da dureza superficial foi realizada no durômetro de bancada do fabricante Time Group Inc. modelo TH600 com esfera de carbeto de tungstênio de diâmetro de $5 \mathrm{~mm}$ com carga aplicada de $750 \mathrm{kgf}$ durante $10 \mathrm{~s}$ [8].

Para realização dos testes de tração utilizou-se uma máquina de tração universal da fabricante EMIC com capacidade de 20 toneladas de carga [6].

Para análise metalográfica as amostras foram cortadas, lixadas e polidas com suspensão de alumina I $\mu$ m e posteriormente atacadas com nital $2 \%$. As microestruturas foram avaliadas em um microscópio óptico (Olympus - mod. UC30) e classificados segundo as normas ASTM A247 e SAE J434 [5,9, I0].

\section{RESULTADOS E DISCUSSÃO}

A composição química das amostras produzidas com a adição dos elementos perlitizantes cobre, antimônio e estanho são apresentados na Tabela 5.

Conforme tabela acima, é possível observar que os percentuais atingidos nas adições dos elementos perlitizantes foram bem próximos aos valores desejados, comprovando a eficiência do procedimento da adição realizado com as quantidades descritas na Tabela 4.

De acordo com a Figura 2, para cada adição de estanho o valor da dureza superficial aumentou como era esperado. Entretanto, a primeira amostra apresenta valores para o desvio padrão abaixo da linha inferior especificada pela norma. As adições seguintes, apresentam os resultados dentro da tolerância.

Em relação as adições de cobre, todas as adições apresentam resultados dentro da especificação. Quanto a dureza $\circ$ aumento foi semelhante ao observado com as

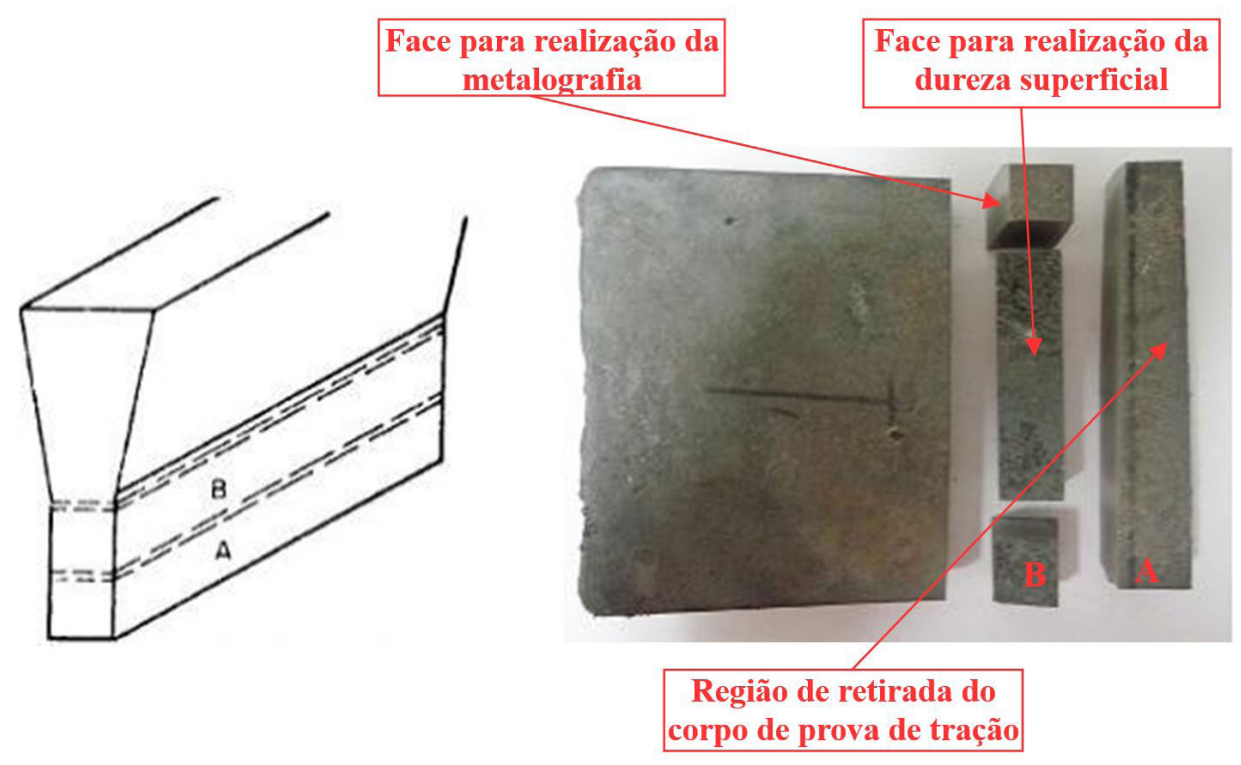

Figura I. Local de retirada dos corpos de provas no bloco Y, A e B locais preferenciais para retirada das amostras. 
Tabela 5. Composição química das amostras com adição dos elementos perlitizantes $\mathrm{Cu}$, Sb e Sn

\begin{tabular}{ccccccccccc}
\hline Elementos & & $\mathbf{C}$ & $\mathbf{S i}$ & $\mathbf{M n}$ & $\mathbf{P}$ & $\mathbf{S}$ & $\mathbf{C u}$ & $\mathbf{S n}$ & $\mathbf{S b}$ & $\mathbf{M g}$ \\
\hline Adição de Cu & $1^{\circ}$ & 3,73 & 2,65 & 0,355 & 0,035 & 0,009 & 0,304 & 0,004 & 0,004 & 0,035 \\
& $2^{\circ}$ & 3,75 & 2,68 & 0,361 & 0,037 & 0,009 & 0,633 & 0,004 & 0,004 & 0,036 \\
\multirow{3}{*}{ Adição de Sb } & $3^{\circ}$ & 3,74 & 2,70 & 0,358 & 0,037 & 0,009 & 0,929 & 0,004 & 0,004 & 0,035 \\
& $1^{\circ}$ & 3,69 & 2,66 & 0,356 & 0,034 & 0,009 & 0,016 & 0,004 & 0,029 & 0,036 \\
& $2^{\circ}$ & 3,70 & 2,72 & 0,364 & 0,036 & 0,009 & 0,015 & 0,004 & 0,060 & 0,039 \\
Adição de Sn & $3^{\circ}$ & 3,72 & 2,68 & 0,372 & 0,032 & 0,009 & 0,019 & 0,004 & 0,090 & 0,037 \\
& $1^{\circ}$ & 3,72 & 2,71 & 0,355 & 0,034 & 0,008 & 0,018 & 0,031 & 0,004 & 0,039 \\
& $2^{\circ}$ & 3,76 & 2,73 & 0,361 & 0,032 & 0,009 & 0,016 & 0,059 & 0,004 & 0,041 \\
& $3^{\circ}$ & 3,70 & 2,69 & 0,358 & 0,035 & 0,009 & 0,020 & 0,091 & 0,004 & 0,038 \\
\hline
\end{tabular}

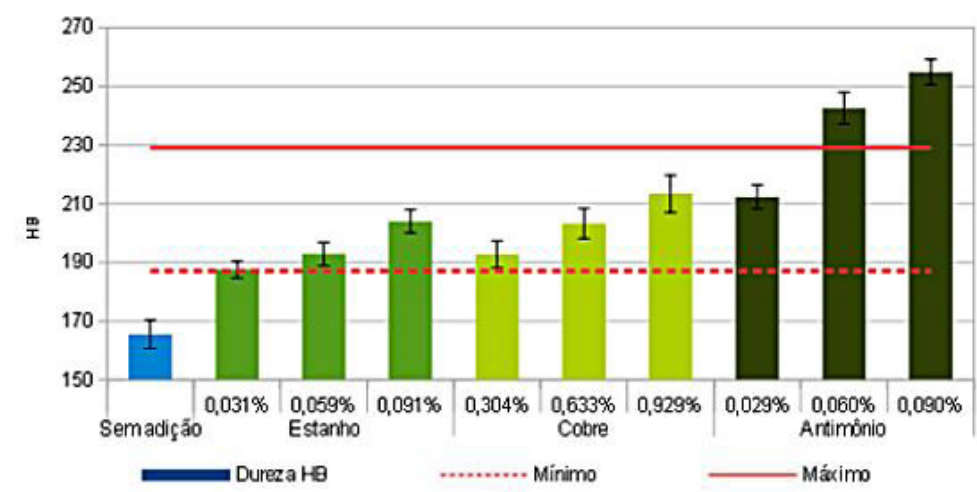

Figura 2. Relação de dureza média das amostras.

adições de estanho, ou seja, ocorreu um pequeno acréscimo de dureza com as adições crescentes de cobre.

Já para a adição de antimônio, somente na primeira adição os valores de dureza ficaram dentro da especificação, na segunda e terceira adição, os valores de dureza ultrapassaram o valor máximo especificado.

$\mathrm{Na}$ Tabela 6 é apresentado o percentual de nodularização, a quantidade de nódulos por $\mathrm{mm}^{2}$, a forma e tamanho da grafita e o percentual de perlita e ferrita presentes na matriz. A amostra sem adição de elementos perlitizantes apresentou uma pequena quantidade de perlita (20\%) e conforme esperado, houve um significativo acréscimo desta microestrutura com adição de cobre, estanho e antimônio [11,12].

As adições de cobre e antimônio se mostraram mais efetivas no aumento de perlita na matriz, sendo o antimônio mais efetivo, pois em sua maior adição, se obteve $98 \%$ de perlita enquanto na maior adição de cobre obtivemos $95 \%$ de perlita, conforme pode ser observado na Figura 3.

O aumento crescente de dureza obtido esta relacionado ao percentual crescente de perlita na matriz metálica, para todos os três elementos adicionados o aumento de dureza está relacionado ao retardo da formação direta de ferrita durante as transformações do estado sólido, este efeito pode ocorrer em função da diminuição da velocidade de difusão do carbono na asutenita, quanto da redução da temperatura eutetóide e da velocidade de transformação $[1|| 2$,$] .$ É importante ressaltar que o cobre também age como elemento grafitizante devido seu efeito de aumentar a diferença entre as temperaturas dos eutético estável e metaestável, porém o estanho e antimônio reduzem a temperatura dos eutéticos, ou seja, dependendo das concentrações podem precipitar carbonetos $[1,2]$.

Em relação ao grau de nodularização observados nas amostras sem adição, em todas as adições de estanho e cobre e ainda a na adição de antimônio com $0,031 \%$, os valores de nodularização ficaram acima de $96 \%$. Entretanto nas adições de 0,060 e 0,090\% de antimônio, ocorreu um decréscimo significativo no grau de nodularização devido a degeneração na morfologia da grafita esferoidal, pelo fato do antimônio criar barreiras de difusão, mas com certa permeabilidade que permite a ancoragem de carbono na superfície da grafita [11,12], ○ grau de nodularização obtido nas maiores adições de antimônio ficaram abaixo do especificado pela norma, ou seja, no mínimo de $80 \%$, conforme se observa na Figura 4. Esta degeneração promove a grafita do tipo "chunky" [13] pelo excesso deste elemento e é acentuada pelo resfriamento lento do fundido $[14,15]$.

Em relação a resistência a tração (Figura 5), a amostra sem adição apresentou um valor médio de resistência à tração de aproximadamente $480 \mathrm{MPa}$, ficando abaixo da 
Tabela 6. Caracterização da microestrutura das amostras

\begin{tabular}{|c|c|c|c|c|c|c|c|}
\hline \multirow{2}{*}{\multicolumn{2}{|c|}{ Condição }} & \multirow{2}{*}{$\begin{array}{c}\text { Nodularização } \\
\text { (\%) }\end{array}$} & \multirow{2}{*}{$\begin{array}{c}\text { Nódulos por } \\
\mathrm{mm}^{2}\end{array}$} & \multirow{2}{*}{$\begin{array}{c}\text { Forma da } \\
\text { grafita }\end{array}$} & \multirow{2}{*}{$\begin{array}{c}\text { Tamanho da } \\
\text { grafita }\end{array}$} & \multicolumn{2}{|c|}{ Matriz (\%) } \\
\hline & & & & & & Perlita & Ferrita \\
\hline Sem adição & & 96,9 & 90 & I (II) & $6(5)$ & 20 & 80 \\
\hline \multirow[t]{3}{*}{ Estanho } & $0,031 \%$ & 96,7 & 82 & I (II) & $6(5)$ & 60 & 40 \\
\hline & $0,059 \%$ & 96,6 & 106 & I (II) & 6 & 70 & 30 \\
\hline & $0,091 \%$ & 96,1 & 116 & I (II) & 6 & 85 & 15 \\
\hline \multirow[t]{3}{*}{ Cobre } & $0,304 \%$ & 96,4 & 95 & I (II) & $6(5)$ & 85 & 15 \\
\hline & $0,633 \%$ & 97,8 & 98 & I (II) & $6(5)$ & 90 & 10 \\
\hline & $0,929 \%$ & 98,0 & 103 & I (II) & 6 & 95 & 5 \\
\hline \multirow[t]{3}{*}{ Antimônio } & $0,029 \%$ & 97,7 & 95 & I (II) & $6(5)$ & 85 & 15 \\
\hline & $0,060 \%$ & 78,8 & 93 & $\mathrm{I}(\mathrm{IV}, \mathrm{II})$ & $6(5)$ & 95 & 5 \\
\hline & $0,090 \%$ & $7 I, 6$ & 71 & I (IV) & $6(5)$ & 98 & 2 \\
\hline
\end{tabular}

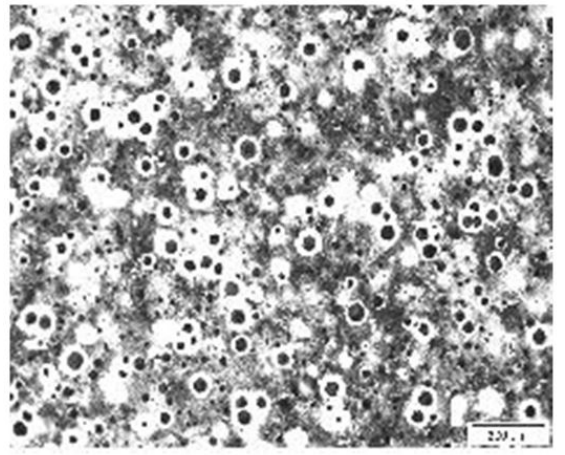

(a)

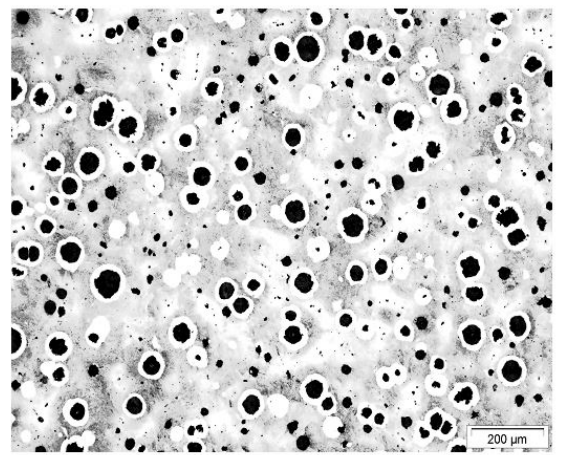

(b)

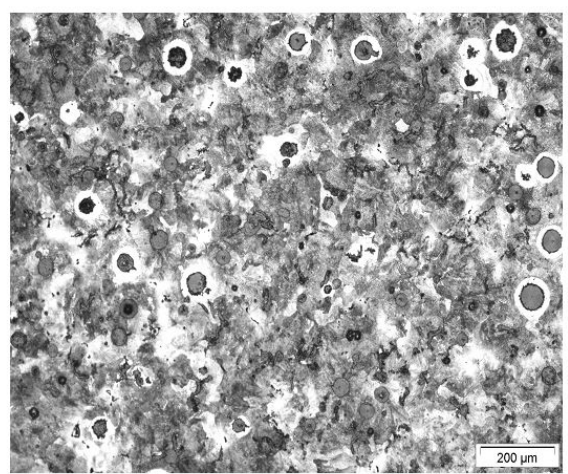

(c)

Figura 3. Metalografias das amostras com maiores adições de estanho (a), cobre (b) a antimônio (c).

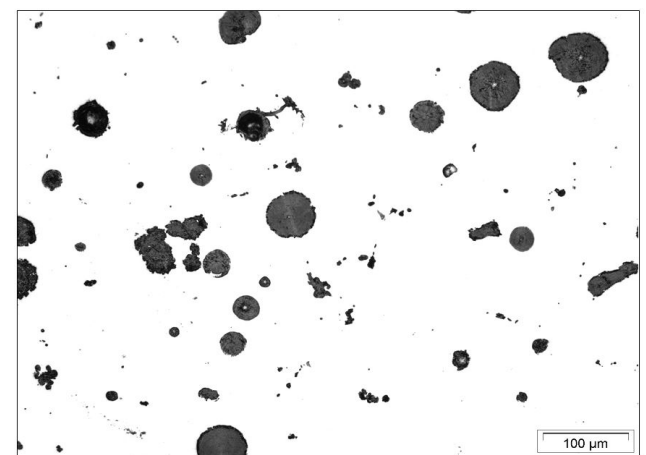

(a)

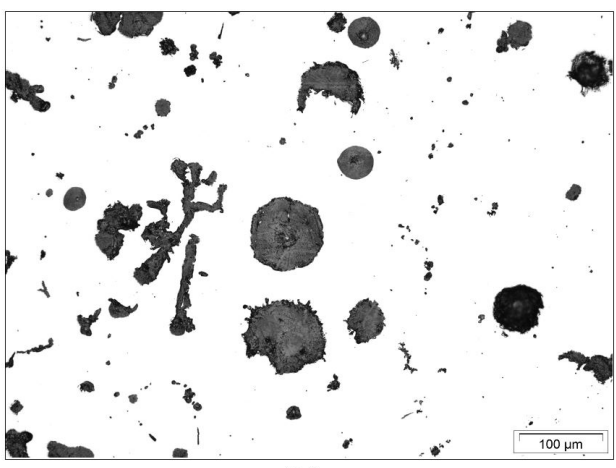

(b)

Figura 4. Metalografias das amostras com adição de 0,060\% (a) e 0,090\% (b) de antimônio. 
especificação mínima da classe D500 que estabelece a resistência mínima de $500 \mathrm{MPa}$.

Nas adições de estanho a eficiência do aumento de resistência é baixa, se comparado com os outros dois elementos perlitizantes mesmo com o aumento de perlita na matriz a cada adição, porém todas as amostras apresentaram valores acima do valor mínimo estabelecido em norma . Em relação ao cobre, o aumento foi crescente conforme o percentual de perlita [16], já o antimônio apresentou esta caracteristica na primeira e segunda adição, na terceira houve uma queda na resistência, atribuido a degeneração da grafita $[I I, I 2]$.

Conforme Figura 6, o alongamento é reduzido devido a redução da fase ferrita e o aumento da perlita que nas amostras de $0,091 \%$ de estanho, $0,060 \%$ e $0,090 \%$ de antimônio os valores ficaram abaixo da especificação.

\section{I Avaliação de Custo}

Com todos os resultados apresentados, verifica-se que as adições de $0,059 \%$ de estanho, todas as adições de cobre e a primeira adição de antimônio, estão com seus resultados dos ensaios dentro da especificação da classe D500. Com isso pode-se comparar as menores adições aprovadas para verificação de custo de cada elemento perlitizante na Tabela 7.

Em relação a quantidade utilizado por quilo de metal, se comparado com o cobre, o estanho reresenta somente 19 , $1 \%$ e antimônio $9,3 \%$ sobre o peso total. No custo, fazendo a mesma comparação, o estanho representa $56,8 \%$ e antimônio $17,0 \%$ sobre o custo total. Verifica-se que o estanho e antimônio comparado com o cobre, apresentam um custo cerca de I,7 e 5,8 vezes menor.

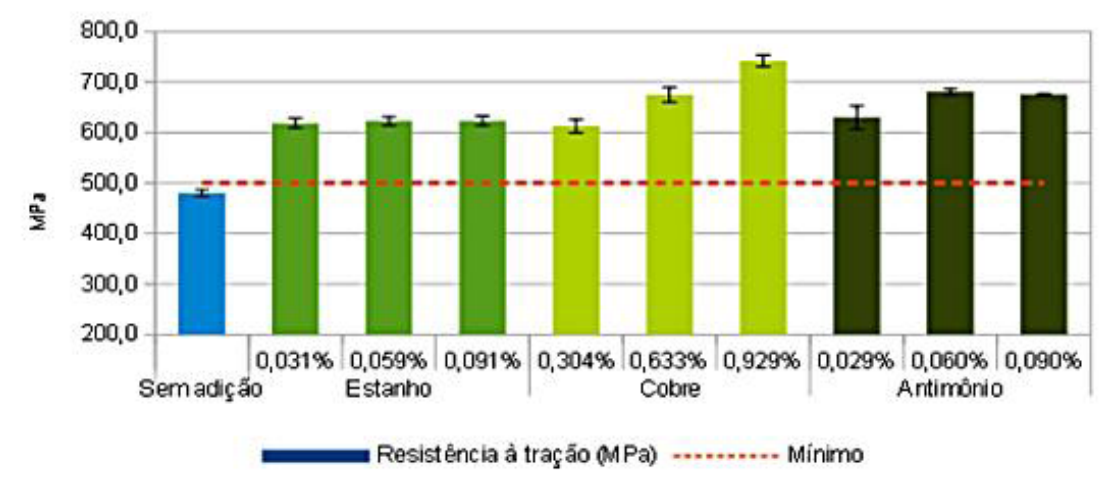

Figura 5. Resultados de resistência à tração.

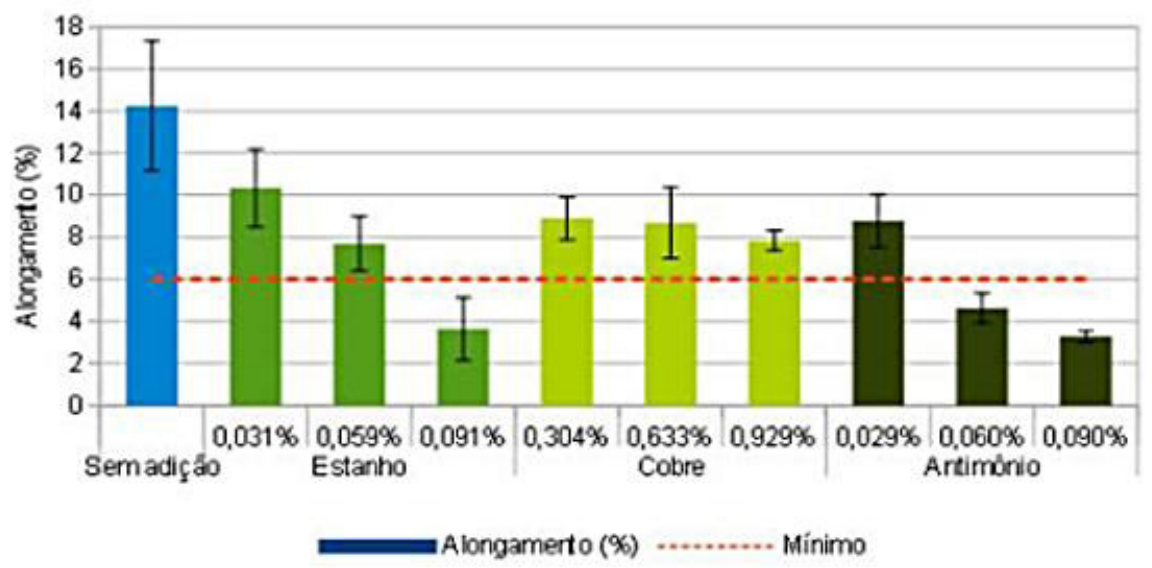

Figura 6. Resultados de alongamento.

Tabela 7. Comparativo entre os elementos perlitizantes

\begin{tabular}{cccccc}
\hline \multicolumn{2}{c}{ Condição } & Peso adicionado por kg & Relação & Custo Médio & Relação \\
\hline Estanho & $0,059 \%$ & 0,000617 & $19,1 \%$ & 0,03392 & $56,8 \%$ \\
Cobre & $0,304 \%$ & 0,003225 & $100,0 \%$ & 0,05966 & $100,0 \%$ \\
Antimônio & $0,029 \%$ & 0,000300 & $9,3 \%$ & 0,01014 & $17,0 \%$ \\
\hline
\end{tabular}




\section{CONCLUSÃO}

Após toda a avaliação feita através de ensaios realizados de acordo com as normas específicas, constata-se que as adições de 0,059\% de estanho, 0,304, 0,633 e 0,929\% de cobre e $0,029 \%$ de antimônio apresentaram os resultados de acordo com a norma SAE J434 da liga D500, sendo possível sua obtenção com essas adições.

Para avaliação de custo, foram utilizados os menores percentuais de cada perlitizante que atingiram as especificações e calculado seu consumo e custo por quilograma. $O$ resultado foi interessante, pois a maioria das fundições que fazem uso de perlitizante para aumento das propriedades mecânicas do ferro fundido nodular, utilizam o elemento cobre. Neste estudo comprova-se que o elemento antimônio é o mais eficiente devido sua menor dosagem, que é a metade da adição de estanho e a décima parte da dosagem do cobre. Em relação ao custo o antimônio é cerca de 3 e 6 vezes menos oneroso que o estanho e cobre respectivamente.

Ainda pode se concluir que para utilização de antimônio e estanho para a obtenção de classes superiores não é possível pois com a adição desses elementos algumas propriedades ficam fora dos parâmetros normativos pois com maiores valores de adições ocorre o mecanismo de degeneração da grafita esferoidal.

$\mathrm{Na}$ utilização do cobre, quase todos os valores dos ensaios permaneceram dentro da classe superior ao estudado, pois como este perlitizante não apresenta nenhum mecanismo de degeneração da grafita é possível utilizar maiores adições.

\section{REFERÊNCIAS}

I Chiaverini V. Aços e ferros fundidos. 7. ed. São Paulo: ABM; 1996.

2 Souza Santos AB, Castello Branco CH. Metalurgia dos ferros fundidos cinzentos e nodulares. I. ed. São Paulo: IPT; 1977.

3 Vilela FJ. Efeito de algumas variáveis de processo na obtenção do ferro fundido nodular ferritico no estado bruto de fundição [dissertação]. São Caetano do Sul: CEUN-EEM; 2010.

4 Fernandes L. Jr. Produção de ferros fundidos cinzentos, nodular e aços em fornos de indução - Aspectos metalúrgicos; 1998.

5 SAE J434. Automotive ductible iron castings. Warrendale: SAE International; 2004.

6 ASTM E8. Standart test method for tension test of metallic materials. West Conshohocken: ASTM Intenational; 2010.

7 ASTM A536. Standard specification for ductile iron castings. West Conshohocken: ASTM International; 1999

8 ASTM E I0. Standart test method for Brinell hardness of metallic materials. West Conshohocken: ASTM International; 2004

9 ASTM A247. Standart test method for evaluating the microestruture of grafite in iron castings. West Conshohocken: ASTM International; 1998

10 NBR 6593-8I. Morfologia de grafita em ferro fundido. NBR. 1981.

II Castelo Branco CH, Souza Santos AB. Efeitos de adições de cobre em ferro fundido nudular hipereutético. Metalurgia. 1975.

12 Koerich LA. Efeito do antimônio na matriz e propriedades mecânicas do ferro fundido nodular. In: Associação Brasileira de Engenharia e Ciências Mecânicas. Anais do $4^{\circ}$ Congresso Brasileiro de Engenharia de Fabricação, 20I0; Campina Grande: ABCM; 2010. p. 10.

13 Liu PC, Li CL, Wu DH, Lopes SEM Jr. Study of chunky graphite in heavy section ductile iron. AFS Transactions. 1983;91:119-126.

I4 Pan EN, Chen CY. Effects of Bi and antimony on graphite structure of heavy-section ductile cast iron. AFS Transactions. 1996; 104:845-858.

I5 Diao XG, Ning ZL, Cao FY, Ren SZ, Sun JF. Effects of antimony addition and section size on formation of chunky graphite in ductile iron. Materials Science and Technology. 201 I;27(4):834-838.

16 Pan EN, Lou MS, Loper CR Jr. Effect of copper, tin and manganese on the eutectoid transformation of graphitized cast iron. AFS Transactions. 1987;87:819-840.

Recebido em: 20 Mar. 2017

Aceito em: 25 Set. 2018 